\title{
Akute Bandverletzung am oberen Sprunggelenk
}

\author{
Roland Biber, Hermann Josef Bail
}

\section{Zusammenfassung}

Akute Bandverletzungen am oberen Sprunggelenk lassen sich einteilen in Läsionen des Außenbands, des Innenbands und der Syndesmose. Ursache der häufigen Außenbandverletzungen sind oft Supinationstraumen. Betroffen ist meist das Lig. fibulotalare anterius, manchmal in Kombination mit dem Lig. fibulocalcaneare. Im Akutfall erfolgt die Therapie meist konservativ mittels Sprunggelenksorthese. Für die operative Versorgung kommt, je nach Rupturlokalisation, die direkte Naht sowie die Re-Insertion transossär oder mittels Fadenanker infrage. Rupturen des Innenbands treten nur sehr selten isoliert auf. Die Therapiewahl hängt daher von den Begleitverletzungen ab. Eine operative Versorgung erfolgt insbesondere dann, wenn das rupturierte Innenband ein Repositionshindernis einer gleichzeitig bestehenden Außenknöchelfraktur oder einer Syndesmosenverletzung darstellt. Innenbandrupturen sind i.d.R. intraligamentär, sodass eine direkte Nahtversorgung möglich ist. Syndesmosenverletzungen treten oftmals gemeinsam mit Sprunggelenksfrakturen (Sonderfall: Maisonneuve-Fraktur) oder mit Innenbandläsionen auf. Sie können aber auch isoliert vorkommen und werden dann leicht übersehen. Eine röntgenologisch nachweisbare Instabilität oder die Kernspintomografie ist dann richtungsweisend. Die Therapie ist i.d.R. operativ mit offener Einstellung und Naht des vorderen Syndesmosenbands sowie der Einbringung fibulotibialer Stellschrauben für etwa 6 Wochen. Knöcherne Syndesmosenausrisse sollten, wenn möglich, osteosynthetisch versorgt werden.

\section{Acute ligament injuries of the ankle}

Acute ligament injuries around the tibiotalar joint may occur as lateral ankle sprains, medial ankle sprains, and syndesmosis sprains (high ankle sprains). The frequent lateral ankle sprains are commonly caused by foot inversion trauma. Isolated disruption of the anterior talofibular ligament (ATFL) occurs more often than the combined rupture of ATFL and CFL (calcaneofibular ligament). Treatment of an acute injury of the lateral ligamentous complex normally is conservative using a brace. Operative reconstruction depends on the place of disruption and may be achieved by direct suture, transosseous sutures, or suture anchors. Isolated medial ankle sprains are very rare. Therefore therapy depends on the associated injuries. Operative treatment is indicated if the disrupted deltoid ligament impedes proper reduction of an ankle fracture or a syndesmosis sprain. Disruptions of the deltoid ligament normally are intrasubstance, allowing for repair by direct suturing. Syndesmosis sprains are often accompanied by ankle fractures (i.e. Maisonneuve lesion) or deltoid ligament ruptures. However there may also be isolated lesions, which can be missed easily. Diagnosis can be made by radiographic detection of instability or by the MRI. The therapy is commonly operative and consists of reduction, anterior inferior tibiofibular ligament reconstruction and application of a fibulo-tibial set screw for 6 weeks. Bony avulsions should be fixed if possible.

\section{Einleitung}

Bandverletzungen am oberen Sprunggelenk sind überaus häufig und kommen insbesondere nach Sportunfällen vor [1]. Oft werden diese Verletzungen unter der verharmlosenden Diagnose „Distorsion“ geführt und nicht mit der gebührenden Aufmerksamkeit bedacht. Der Bandapparat im Sprunggelenksbereich weist eine komplexe Anatomie auf, die für das Verständnis dieser Verletzungen von großer Bedeutung ist. Der vorliegende

OP-JOURNAL 2014; 30: 92-97

(c) Georg Thieme Verlag KG Stuttgart · New York DOI http://dx.doi.org/10.1055/s-0034-1383179
Artikel konzentriert sich auf die häufigen akuten Bandverletzungen im Bereich des oberen Sprunggelenks. Entsprechende Verletzungen des unteren Sprunggelenks mit subtalarer Instabilität sind wesentlich seltener [11] und werden hier, ebenso wie die Problematik der chronischen Instabilität, nicht behandelt. Akute Bandverletzungen des oberen Sprunggelenks betreffen den Außenbandapparat, das Innenband und/oder die Syndesmose.

\section{Außenbandverletzungen}

Akute Rupturen des Außenbands sind überaus häufig und treten oft als Sportverletzung auf, insbesondere bei Basket- ball und Fußball. Grundsätzlich sind sie eine Domäne der konservativen Therapie. Jedoch sollten derartige Verletzungen keinesfalls unterschätzt werden: In bis $\mathrm{zu} 32 \%$ der Fälle verbleiben chronische Schmerzen, Schwellungszustände oder wiederholte Distorsionen; in bis zu 19\% Instabilitätsprobleme mit rezidivierendem Umknicken [5, 9].

\section{Anatomie und Biomechanik}

Der Außenbandkomplex des Sprunggelenks umfasst 3 Anteile (vgl. Abb. 1):

- Lig. fibulotalare anterius (ATFL): Verläuft von der anterioren Fibulaspitze zum Talus, wo es unmittelbar anterior der Gelenkfacette ansetzt. Das Band ist 


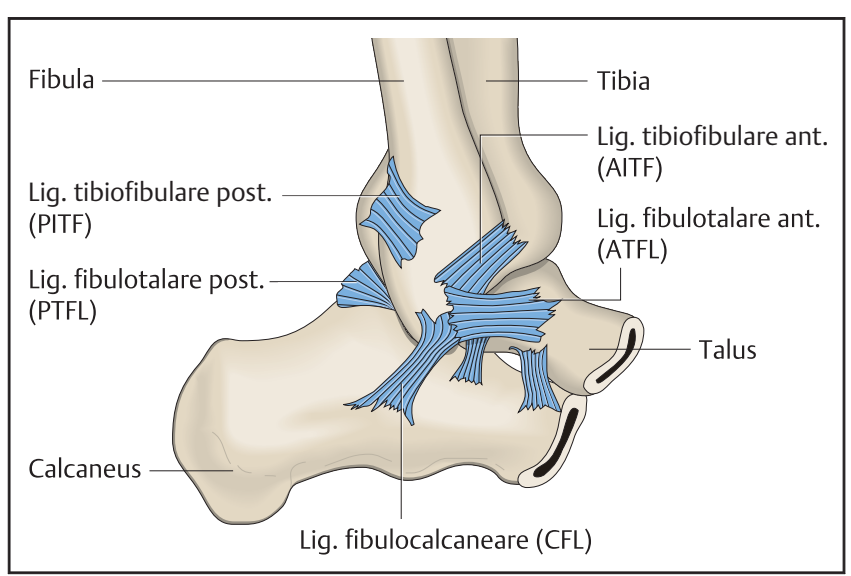

Abb. 1 Ansicht von lateral auf den Außenbandapparat und Teile der Syndesmose.

15-20 mm lang, 6-8 $\mathrm{mm}$ breit sowie $2 \mathrm{~mm}$ dick [19]. Meist besteht es aus 2 Anteilen, zwischen denen Gefäße hindurchtreten. Das Band geht in die vordere Gelenkkapsel über.

- Das ATFL ist in Dorsalextension locker und spannt sich bei Plantarflexion an. Es verhindert in erster Linie eine Innenrotation des Talus in der Sprunggelenksgabel; in Plantarflexionsstellung wirkt es auch einer Adduktion entgegen $[2,10,17,21]$.

- Lig. fibulocalcaneare (CFL): Entspringt distal des ATFL und zieht schräg nach dorsomedial zum Kalkaneus. Das Band ist 2-3 cm lang, 4-8 $\mathrm{mm}$ breit und 3$5 \mathrm{~mm}$ dick. Es geht in die Peronealsehnenscheide über [19].

- Das CFL ist in Dorsalextension angespannt und bei Plantarflexion locker. Das Band wirkt einer Adduktion des Fußes entgegen $[2,10,17,21]$.

- Lig. fibulotalare posterius (PTFL): Von seinem Ursprung an der dorsalen Innenseite des Malleolus lateralis verläuft es zum dorsalen Talus, wo es breitflächig ansetzt. Das Band ist $3 \mathrm{~cm}$ lang, $5 \mathrm{~mm}$ breit und $5-8 \mathrm{~mm}$ dick [19]. In Dorsalextension ist es angespannt und limitiert diese zusammen mit dem Innenband. Ferner wirkt es einer Außenrotation des Talus entgegen $[2,10,17,21]$.

Diese anatomischen Verhältnisse führen dazu, dass es, je nach Unfallmechanismus, zu unterschiedlichen Verletzungsmustern kommen kann [17]:

- Forcierte Innenrotation führt zur Ruptur des ATFL und nachfolgend des PTFL.

- Übermäßige Dorsalextension führt zur Ruptur des PTFL.

- Übermäßige Adduktion führt in neutraler und dorsalextendierter Stellung eher zur CFL-Ruptur, in plantarflektierter Stellung eher zur ATFL-Ruptur.

\section{Verletzungsarten}

Die isolierte ATFL-Ruptur gilt als die mit Abstand häufigste Verletzung [1]. Meist sind diese Rupturen intraligamentär, jedoch kommen auch knöcherne Bandausrisse an Fibula oder Talus vor. Am zweithäufigsten sind Kombinationsrupturen von ATFL und CFL, die ebenfalls meist intraligamentär auftreten. Isolierte CFL-Rupturen sind selten und bedingen dann möglicherweise auch eine Instabilität des unteren Sprunggelenks $[4,6,11]$. Die Ruptur aller 3 Bänder des Außenbandkomplexes ist sehr selten und Rupturen des PTFL mit oder ohne CFL sind eine Rarität.

Außenbandrupturen können mit zahlreichen weiteren Verletzungen einhergehen, nach denen in jedem Fall gefahndet werden sollte. Hierunter fallen komplette oder partielle Rupturen der Peronealsehnen, Innenbandläsionen, osteochondrale Frakturen des Talus, Syndesmosenverletzungen, Frakturen an der Metatarsale-V-Basis, sowie kalkaneokuboidale Impressionsfrakturen [1].

Auch die Fraktur des Processus lateralis tali, der sog. „Snowboarder's ankle“, führt zu Schmerzen im lateralen Sprunggelenksbereich [12]. Grundsätzlich können akute Bandverletzungen auch mit Nervenverletzungen einhergehen, welche eine Störung der Propriozeption bedingen und so einer (funktionellen) Instabilität zusätzlich Vorschub leisten [7, 18].

\section{Diagnostik}

In der Anamnese wird meist ein Umknicktrauma im Sinne einer Supination angegeben, wobei nur manche Patienten den stattgehabten Mechanismus bzw. die Gelenkstellung zum Zeitpunkt des
Traumas genau beschreiben können. Oftmals handelt es sich nicht um das Erstereignis, sodass eine chronische Instabilität zu vermuten ist. Klinisch zeigt sich eine schmerzhafte Schwellung und oftmals ein Hämatom im Außenbandbereich (Abb. 2). Nur beim ganz frischen Trauma lassen sich Anteile des Außenbands bei subtiler manueller Untersuchung zuverlässig unterscheiden. Meist fällt eine Bewegungseinschränkung für Extension/Flexion sowie insbesondere für die Supination auf. Das vordere Schubladenzeichen provoziert Schmerzen bei ATFL-Teilrupturen, kann aber bei Komplettrupturen auch schmerzarm durchzuführen sein. Wichtig für die Stabilitätsbeurteilung ist es, den Schubladentest mit gebeugtem Kniegelenk durchzuführen (Entspannung der Achillessehne). Immer sollte auch die Syndesmose überprüft werden.

Röntgenaufnahmen sind nach Umknicktraumen nicht immer notwendig; ein Unterlassen setzt allerdings einige Erfahrung in der körperlichen Untersuchung voraus. Eine Hilfestellung zur Indikationsstellung für die konventionelle Röntgenuntersuchung liefern die Ottawa Ankle Rules [20]; zusammengefasst empfehlen diese eine Röntgenuntersuchung bei Schmerzen über dem Außen- oder Innenknöchel, dem Metatarsale- $V$ sowie bei Unfähigkeit zur Gewichtsbelastung. Röntgenbilder sollten in mind. 2 Ebenen angefertigt werden, wobei bei der a.-p.Aufnahme für eine gute Einstellung der Sprunggelenksgabel das Bein ca. $20^{\circ}$ nach innen gedreht werden sollte („mortise view“).

Die Zuverlässigkeit wie auch die konkrete Durchführung gehaltener Röntgenaufnahmen sind insbesondere wegen der großen Variabilität der Normalbefunde umstritten. Eine Analgesie scheint die Wertigkeit von Stressaufnahmen zu erhöhen. In Neutralstellung testet der Supinationsstress eher das CFL, in Plantarflexionsstellung dagegen das ATFL. Beide Stellungen sowie auch das vordere Schubladenzeichen sollten angewendet werden. Die meisten Autoren sehen eine Talusverkippung von $>15^{\circ}$ oder einen Talusvorschub von $>5 \mathrm{~mm}$ als pathologisch an [1].

Wenngleich in geübter Hand die Ultraschalldiagnostik Außenbandverletzungen sehr zuverlässig feststellen kann, muss derzeit das MRT als Goldstandard der Diagnostik bezeichnet werden. Es bietet den Vorteil, ggf. zusätzliche int- 
raartikuläre Verletzungen, wie z.B. osteochondrale Frakturen, zuverlässig zu erkennen.

\section{Einteilung}

Bisher hat sich keine Klassifikation vollständig durchgesetzt. Häufig finden sich 3-stufige Gradeinteilungen, die sich entweder an der Zahl der betroffenen Bandanteile (ATFL, CFL, PTFL) oder am Ausmaß der Läsion (Zerrung, Teilruptur, vollständige Ruptur) orientieren.

\section{Therapie}

Bandverletzungen werden in den allermeisten Fällen konservativ behandelt, dies gilt insbesondere für die Fälle ohne Nachweis einer Instabilität. Verschiedene Methoden der Ruhigstellung kommen zur Anwendung, diese reichen von der Gipsruhigstellung über Sprunggelenksorthesen bis hin zu Tapingverfahren. Die empfohlene Behandlungsdauer liegt bei 6 Wochen; eine funktionelle Nachbehandlung mit zügigem Übergang zur vollen Gewichtsbelastung ist dabei der kompletten Ruhigstellung überlegen. Entscheidend für den Therapieerfolg ist selbstverständlich, dass die Supination während dieser Zeit limitiert wird. $\mathrm{Zu}$ beachten ist aber auch, dass es durch Dorsalextension zu einer Annäherung der Rupturenden des ATFL kommt, was möglicherweise eine stabile Ausheilung fördert. Nach der Initialphase mit Hochlagern, Kühlung, Schonung und Kompression spielen Bewegungs- und Propriozeptionsübungen eine wichtige Rolle.

Konservative und operative Therapie erbringen ähnliche funktionelle Ergebnisse, sodass wegen des zusätzlichen Risikos einer Operation von dieser meist Abstand genommen wird. Eine OP-Indikation wird allenfalls in den Fällen gesehen, bei denen eine Instabilität bei kombinierter Ruptur von mind. ATFL und CFL vorliegt oder weitere Verletzungen vorliegen, die einer operativen Behandlung bedürfen (offene Verletzungen, Frakturen). Bei einer operativen Versorgung einer Außenbandläsion liegen der N. peronaeus superficialis und der $\mathrm{N}$. suralis subkutan im OP-Bereich und sollten möglichst geschont werden (postoperative Sensibilitätsstörungen in 7-19\% [1]). Intraoperative Stresstests erleichtern das Auffinden der Rupturenden. Die meist intraligamentären Rupturen werden mit resorbierbarem Nahtmaterial genäht; knöcherne Ausrisse, je nach Situation,

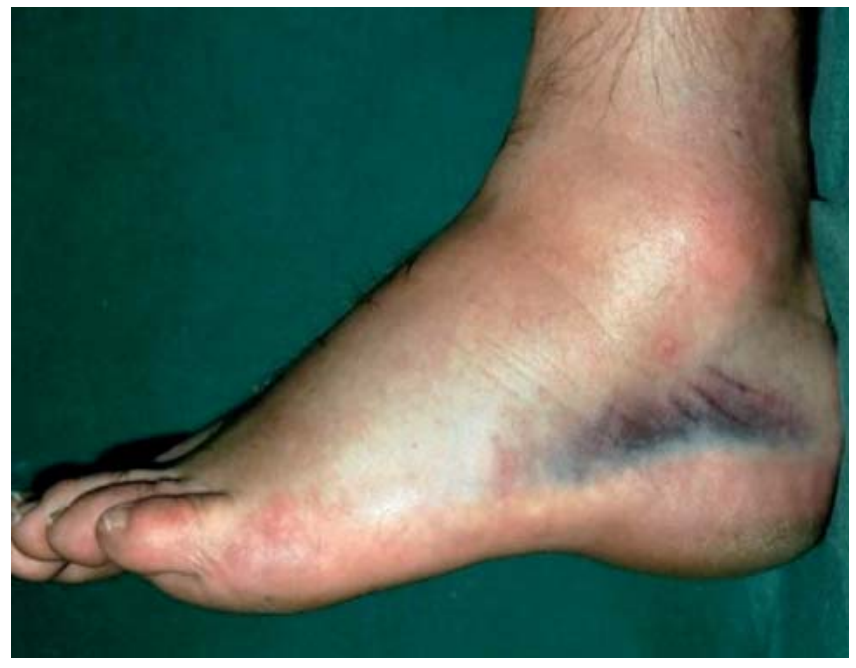

Abb. 2 Klinische Zeichen einer Außenbandruptur: Schmerzhafte Schwellung und Hämatom unterhalb des Außenknöchels.

mittels Schraubenosteosynthesen, transossären Nähten oder Knochenankern refixiert. Bei guter Rekonstruktion von ATFL und CFL muss eine Naht des evtl. ebenfalls rupturierten PTFL nicht erzwungen werden, da dies das funktionelle Ergebnis nicht zu beeinträchtigen scheint. Auch eine rupturierte Peronealsehnenscheide und die vordere Gelenkkapsel werden, soweit erreichbar, vernäht. Die Nähte werden dabei zunächst vorgelegt und schließlich in Neutralstellung des Fußes von dorsal nach ventral geknüpft. Die Nachbehandlung erfolgt bspw. für 4 Wochen unter Vollbelastung in einem Walker, nachfolgend für weitere 4 Wochen mit einer Sprunggelenksorthese.

\section{Innenbandverletzungen}

Isolierte Innenbandverletzungen nach Umknicktrauma sind selten; meist liegen sie in Kombination mit Frakturen oder Außenbandläsionen vor.

\section{Anatomie und Biomechanik}

Das Innenband oder Lig. deltoideum besteht aus einem oberflächlichem und einem tiefen Anteil. Beim oberflächlichen Anteil gehen eine Pars tibionavicularis, eine Pars tibiocalcanea (TCL) und eine Pars tibiotalaris posterior (PTTL) ineinander über. Der tiefe Anteil besteht hauptsächlich aus der Pars tibiotalaris anterior. Insgesamt zieht das Band somit fächerförmig vom Innenknöchel nach unten.

Das TCL wirkt in erster Linie einer forcierten Abduktion entgegen. Der tiefe Anteil verhindert die Außenrotation in dorsalextendierter Stellung. Ein vorderes
Schubladenphänomen entsteht durch eine alleinige Läsion des Deltabands nicht, jedoch führt diese zu einer Verstärkung des Vorschubs bei Ruptur des Außenbands. Einer Lateralverschiebung des Talus wirkt zunächst die intakte Fibula entgegen; bei Verlust dieses Stabilisators (z.B. Fibulafraktur) erlaubt das intakte Deltaband eine Lateralverschiebung des Talus um maximal ca. $3 \mathrm{~mm}$, bevor es rupturiert. Ein mediales Aufklappen des Tibiotalargelenks ist beweisend für eine Komplettruptur aller Anteile des Deltabands. Rupturen befinden sich meist intraligamentär nahe des distalen Bandsansatzes.

\section{Verletzungsarten}

Typisch ist ein Eversions- oder Pronationstrauma des Sprunggelenks. Isolierte Innenbandverletzungen sind dabei eher selten und kommen wohl in etwa 4\% der Fälle vor [5]. Meist finden sich Innenbandverletzungen in Kombination mit Frakturen, Außenband- oder Syndesmosenrupturen.

\section{Diagnostik}

Die körperliche Untersuchung sollte neben der Erfassung von Schmerzen, Schwellung und Hämatomen im Innenbandbereich auch die Fibula in voller Länge sowie die Außenbänder und die Syndesmose erfassen. Läsionen der Sehnen des M. tibialis posterior, des M. flexor digitorum longus sowie des M. flexor hallucis longus sollten durch entsprechende Funktionstests ausgeschlossen werden. Nervenschäden können durch Zug am N. tibialis und N. saphenus entstehen. Eine mediale Aufklappbarkeit kann ebenfalls klinisch getestet werden; 
dies kann aber schmerzbedingt eingeschränkt sein und wäre überdies nur bei Komplettrupturen auffällig.

Auch röntgenologisch zeigt sich eine mediale Aufklappung nur bei einer vollständigen Ruptur. Das MRT eignet sich grundsätzlich zur Darstellung des Innenbands. Es ist aber zu beachten, dass koronare Schichten die Pars tibionavicularis und die Pars tibiotalaris anterior in einer Plantarflexion von $40-50^{\circ}$ in ihrem Verlauf darstellen, während TCL und PTTL sich am besten in koronaren Schichten bei $10-20^{\circ}$ Dorsalextensionsstellung abbilden.

\section{Einteilung}

Bisher hat sich keine Klassifikation durchgesetzt, zumal die therapeutischen Konsequenzen stark von den Begleitverletzungen abhängen. Letztlich wird oftmals analog zum Außenband eine 3-stufige Gradeinteilung verwendet (Grad I: Zerrung, Grad II: Teilruptur, Grad III: Komplettruptur). Hintermann unterscheidet darüber hinaus nach Lokalisation proximale Rupturen bzw. knöcherne Ausrisse (Typ I), Rupturen in Bandmitte (Typ II) und distale Rupturen (Typ III), wobei letztere am häufigsten vorkommen [8].

\section{Therapie}

Die Therapieform hängt von den vorliegenden Begleitverletzungen ab. Bei Außenknöchelfrakturen oder Syndesmosenverletzungen ist eine operative Versorgung der Innenbandläsion per se nicht zwingend erforderlich. Jedoch kann ein eingeschlagenes Innenband ein Repositionshindernis darstellen, wodurch dann doch eine Innenbandrevision notwendig wird. In diesem Fall sollte das Band dann genäht werden. Isolierte Innenbandrupturen können konservativ behandelt werden. Dies erfolgt meist mittels Orthese oder Gehgips für 6-8 Wochen. Die operative Versorgung erfolgt meist durch eine Hautinzision, die von der dorsalen Innenknöchelspitze schräg nach distal in Richtung des Talonavikulargelenks geführt wird. Das Gelenk wird durch die Ruptur soweit wie möglich inspiziert und ausgespült. Falls die tiefen und oberflächlichen Bandanteile identifizierbar sind, können sie separat genäht werden. Bei Avulsionsverletzungen können Nahtanker zum Einsatz kommen. Auch nach operativer Versorgung richtet sich die Nachbehandlung nach den Begleitverletzungen; alleine vonseiten der Bandverletzung wäre eine Behandlung mittels Orthese i.d.R. ausreichend.

\section{Syndesmosenverletzungen}

Die Syndesmosenverletzung wird in der Literatur meist im Zusammenhang mit Sprunggelenksfrakturen vom Typ Weber $C$ bzw. den sog. Maisonneuve-Frakturen diskutiert, jedoch kommen Rupturen des Syndesmosenbands auch isoliert vor und machen bis zu 18\% der Bandverletzungen am Sprunggelenk aus.

Syndesmosenverletzungen können leicht übersehen werden, da die Elastizität der Gelenkstrukturen im unbelasteten Zustand oft zur spontanen Reposition im distalen Tibiofibulargelenk führt und in der Röntgenaufnahme dann keine Diastase zwischen diesen beiden Knochen zu erkennen ist [1].

Im Rahmen von Kontaktsportarten ist die Inzidenz deutlich erhöht; röntgenologisch sichtbare Ossifikationen im Syndesmosenbereich werden als Spätfolge solcher Verletzungen interpretiert.

\section{Anatomie und Biomechanik}

Tibia und Fibula werden durch mehrere Bandstrukturen zusammengehalten, die man unter dem Oberbegriff der Syndesmose zusammenfasst (vgl. auch Abb. 1):

- Membrana interossea: Verbindet Tibia und Fibula über praktisch deren gesamte Länge.

- Lig. tibiofibulare anterius (AITF): Verläuft $45^{\circ}$ schräg absteigend vom Tubercule de Chaput-Tillaux an der anterolateralen Tibia zur Ventralseite des Außenknöchels. Das Band ist $2-3 \mathrm{~cm}$ lang und $20 \mathrm{~mm}$ breit. Der distale Anteil kann arthroskopisch eingesehen werden und eine Impingement-Problematik verursachen [17]. Der Anteil an der Syndesmosenstabilität wird mit 35\% eingeschätzt [14].

- Lig. interosseum: Das Band verbindet Tibia und Fibula in der Inzisur. Es ist $0,5-2 \mathrm{~cm}$ oberhalb des Plafonds gelegen und setzt sich nach kranial in die Membrana interossea fort [15]. Der Anteil an der Syndesmosenstabilität wird mit $22 \%$ eingeschätzt [14].

- Lig. tibiofibulare posterius (PITF): Dieses besteht aus einem oberflächlichen und einem tiefen Anteil. Von der dorsalen Tibia verläuft es zur Dorsalseite des Außenknöchels. Bei einer Länge von $30 \mathrm{~mm}$ und einer Breite von $20 \mathrm{~mm}$ ist das Band etwa $5 \mathrm{~mm}$ dick [17]. Der Anteil an der Syndesmosen- stabilität wird mit 9\% (oberflächlicher Anteil) bzw. 33\% (tiefer Anteil) eingeschätzt [14].

Die Syndesmose stabilisiert die Sprunggelenksgabel, ermöglicht aber gleichzeitig eine gewisse Elastizität. So weichen Tibia und Fibula bei Dorsalextension im oberen Sprunggelenk etwa 1,5 mm auseinander und auch begrenzte Rotationsbewegungen zwischen Tibia und Talus spielen für den physiologischen Bewegungsablauf eine wichtige Rolle. Bei Durchtrennung des AITF wird eine $\mathrm{Zu}$ nahme des tibiofibularen Abstands zwischen 4 und $12 \mathrm{~mm}$ berichtet; Diskontinuitäten des Lig. interosseum und des PITF erhöhen dies um jeweils mehrere Millimeter [1,14].

\section{Verletzungsarten}

Nach unserem Kenntnisstand ist es bisher im Biomechaniklabor nicht gelungen, durch äußerliche Krafteinwirkung isolierte Syndesmosenverletzungen ohne knöcherne Beteiligung $\mathrm{zu}$ erzeugen. Dennoch gilt eine forcierte Außenrotation des Fußes (v. a. in dorsalextendierter Stellung) als der wichtigste ursächliche Pathomechanismus, wobei zunächst das AITF reißt und die weiteren Strukturen nur bei fortgesetzter Gewalt betroffen sind [1]. Das PITF ist nur sehr selten betroffen. Bei einer forcierten Abduktion kann es zur Syndesmosenruptur in Verbindung mit einer Innenband- oder Innenknöchelläsion kommen.

\section{Diagnostik}

Bei frühzeitiger Untersuchung können Schmerz und Schwellung gut auf den Bereich des AITF eingegrenzt werden. Insbesondere bei Unfallmechanismen mit Abduktionskomponente sollte auch das Innenband und der Innenknöchel sorgfältig untersucht werden. Wegen der Möglichkeit einer Maisonneuve-Verletzung ist die Fibula auf deren gesamter Länge zu palpieren. Mehrere klinische Tests zur Prüfung der Syndesmosenstabilität wurden vorgeschlagen [1], sind jedoch begrenzt zuverlässig:

- Squeeze-Test nach Pillings: Tibiofibulare Kompression oberhalb der Unterschenkelmitte führt zu Schmerzen im Syndesmosenbereich aufgrund der dadurch provozierten Verschiebung zwischen Tibia und Fibula

- Cotton-Test: Vermehrte mediolaterale Verschiebung des Fußes gegen den fixierten Unterschenkel im Seitenvergleich 


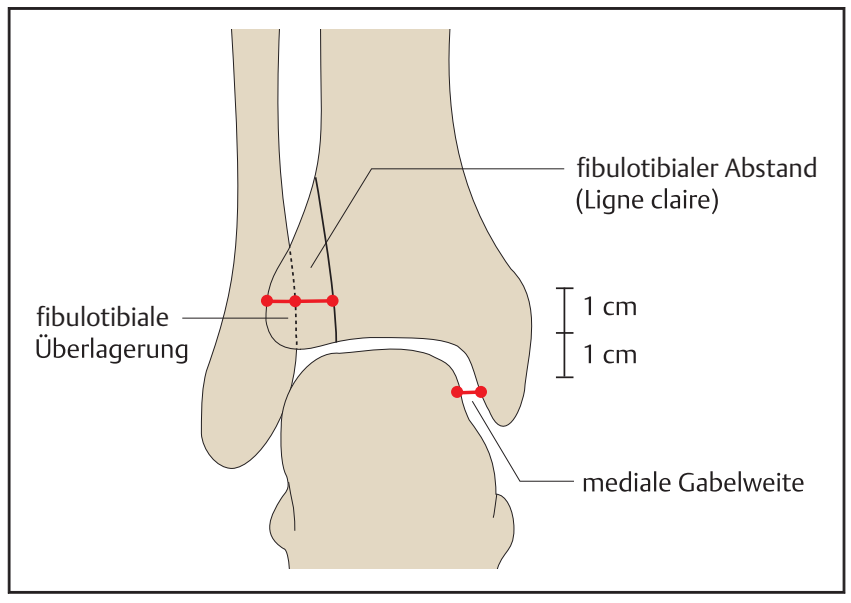

- Außenrotationstest: Außenrotation des Fußes gegen den fixierten Unterschenkel bei gebeugtem Kniegelenk führt zu Schmerzen im Syndesmosenbereich

In aller Regel sollte jeder Verdacht auf eine Syndesmosenverletzung zu einer radiologischen Diagnostik führen. An deren Anfang stehen konventionelle Röntgenaufnahmen in 2 Ebenen, die u.a. knöcherne Syndesmosenausrisse nachweisen können. In 10-50\% aller Syndesmosenverletzungen liegen knöcherne Avulsionen im Bereich des Tubercule de Chaput (Tillaux-Fraktur) oder seltener des Außenknöchels (Wagstaffe-Fraktur) vor [1]. Auf der a.-p.-Aufnahme kann weiterhin die korrekte Lagebeziehung zwischen Tibia, Fibula und Talus durch Analyse bestimmter Abstände festgestellt werden (vgl. Abb. 3):

- fibulotibialer Abstand $1 \mathrm{~cm}$ oberhalb des Plafonds („ligne claire“ nach Chaput): pathologisch wenn $>5 \mathrm{~mm}$

- fibulotibiale Überlagerung $1 \mathrm{~cm}$ oberhalb des Plafonds: pathologisch wenn $<6 \mathrm{~mm}$

- Abstand zwischen Innenknöchel und Talus $1 \mathrm{~cm}$ unterhalb des Plafonds (mediale Gabelweite bzw. „medial clear space“): pathologisch wenn $>4 \mathrm{~mm}$

Auch bei Vorliegen einer Syndesmosenruptur können konventionelle Röntgenuntersuchungen unauffällig bleiben, sodass bei entsprechendem Verdacht Stressaufnahmen indiziert sind. Diese werden in Außenrotation und Abduktion des Fußes durchgeführt. Neben einer Aufweitung der Sprunggelenksgabel in der a.-p.-Projektion kann es auch zu einer Dorsalverschiebung der Fibula in der seitlichen Ansicht kommen. Auch die Wertigkeit der gehaltenen Aufnahmen ist umstritten, sodass im Zweifel
Abb. 3 Parameter zur Abschätzung der Stellung des distalen Tibiofibulargelenks im konventionellen Röntgen (a.-p.). Für die Analyse der Rotationseinstellung ist eine Schnittbildgebung erforderlich.

eine MRT-Untersuchung angeschlossen werden sollte. Diese gilt mit einer Sensitivität von bis zu 100\% bei einer Spezifität von ca. 93\% als Goldstandard [13].

\section{Einteilung}

International am verbreitetsten ist wohl die Klassifikation nach Edwards und DeLee [3], welche insbesondere die latente, nur auf Stressaufnahmen erkennbare tibiofibulare Diastase von der bereits ohne äußere Einwirkung manifesten Fehlstellung unterscheidet.

\section{Therapie}

Wie üblich bestehen die Primärmaßnahmen aus Schonung, Kühlung, Hochlagerung, Kompression und Ruhigstellung. Eine konservative Therapie mit Knöchelorthese für 6 Wochen ist nur bei isolierten AITF-Verletzungen ohne Instabilität und ohne knöcherne Beteiligung möglich. Ansonsten ist die Therapie in aller Regel operativ und umfasst 2 Komponenten:

- Reposition und Rekonstruktion des AITF: Längsinzision vor dem Außenknöchel im Bereich der vorderen Syndesmose, wobei der N. peronaeus superficialis das OP-Gebiet kreuzt und geschont werden sollte. Die Reposition kann mit einer großen spitzen Zange erfolgen, die über der Sprunggelenksgabel angebracht wird; hierbei ist insbesondere auch auf die Rotationseinstellung zu achten. Schwierigkeiten bei der korrekten Einstellung sind häufig durch ein eingeschlagenes Innenband bedingt, welches in diesem Fall revidiert werden muss. Eine Fibulaosteotomie ist sehr selten und praktisch nur bei veralteten Fällen nötig. Die Syndesmose selbst wird direkt genäht oder transossär refixiert (z.B.

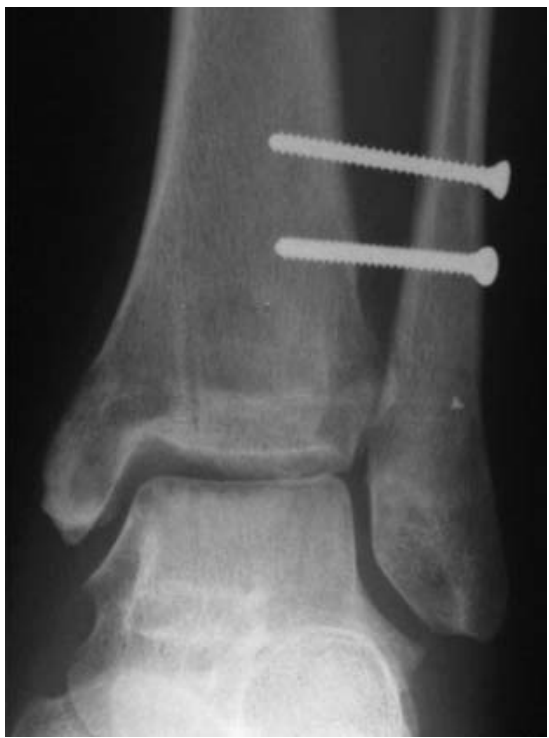

Abb. 4 Beispiel einer fehlgeschlagenen Versorgung: Erhöhter fibulotibialer Abstand bei verminderter fibulotibialer Überlagerung in der a.-p.-Projektion. Bei der Primäroperation waren nur Stellschrauben eingebracht worden. Eine offene Rekonstruktion des vorderen Syndesmosenbands war jedoch unterblieben und die geschlossene Reposition des distalen Tibiofibulargelenks gelang nicht vollständig. Bei der Revisionsoperation zeigte sich als Repositionshindernis eine eingeschlagene Innenbandruptur.

Knochenanker), bei knöchernen Ausrissen kommen Osteosynthesen mit Minischrauben infrage.

- Stellschraube: Stellschrauben werden ca. $2 \mathrm{~cm}$ oberhalb der Syndesmose ca. $30^{\circ}$ von dorsal kommend fibulotibial eingebracht, wobei in jedem Fall 2 Kortikales der Fibula zu erfassen sind. Es besteht jedoch kein Konsens darüber, ob 1 oder 2 Stellschrauben zu verwenden sind oder ob diese in der Tibia mono- oder bikortikal verankert werden sollten.

Die Kontrolle der korrekten tibiofibularen Positionierung sollte im Zweifel postoperativ mittels CT oder bereits intraoperativ durch 3-D-Bildgebung überprüft werden, um verbliebene Fehlstellungen sicher auszuschließen (vgl. Abb.4). Bezüglich der Nachbehandlung bestehen verschiedene Schemata. $\mathrm{Zu}$ meist sehen diese die Verwendung eines Walkers sowie eine Teilbelastung von ca. $20 \mathrm{~kg}$ vor. Bei frühzeitiger Vollbelastung besteht die Gefahr der Schraubenlockerung bzw. des Schraubenbruchs. Die Entfernung der Stellschrauben erfolgt üblicherweise nach ca. 6 Wochen. 


\section{Fazit}

Bandverletzungen des oberen Sprunggelenks betreffen das Außenband, das Innenband und/oder die Syndesmose. Die zugrunde liegende Anatomie ist komplex. Außenbandrupturen sind häufig und betreffen meist das Lig. fibulotalare anterius sowie das Lig. fibulocalcaneare. Bei im unkomplizierten Fall vergleichbarem funktionellem Outcome birgt die operative Therapie zusätzliche Risiken, sodass die konservative Therapie derzeit bevorzugt wird. Innenbandläsionen kommen meist in Kombination mit anderen Verletzungen vor und sollen dann operativ versorgt werden, wenn sie ein Repositionshindernis darstellen. Syndesmosenrupturen sind dagegen eine Domäne der operativen Therapie; empfohlen wird die Rekonstruktion der vorderen Syndesmose sowie die Sicherung mittels Stellschraube(n) für etwa 6 Wochen.

\section{Literatur}

${ }^{1}$ Clanton TO, McGarvey W. Athletic Injuries to the Soft Tissues of the Foot and Ankle. In Coughlin MJ, Mann RA, Saltzman CL, eds. Surgery of the Foot and Ankle. Philadelphia: Mosby/Elsevier; 2007: 1451-1489

2 Colville MR, Marder RA, Boyle IJ et al. Strain measurement in lateral ankle ligaments. Am J Sports Med 1990; 18: 196-200
${ }^{3}$ Edwards GS, DeLee JC. Ankle diastasis without fracture. Foot Ankle 1984; 4: 305-312

${ }^{4}$ Francillon MR. Distorsio pedis with an isolated lesion of the ligamentum calcaneo-fibulare. Acta Orthop Scand 1962; 32: 469475

${ }^{5}$ Gerber JP, Williams GN, Scoville CR et al. Persistent disability with ankle sprains: a prospective examination of an athletic population. Foot Ankle Int 1998; 19: 653-660

${ }^{6}$ Heilman AE, Braly WG, Bishop JO et al. An anatomic study of subtalar instability. Foot Ankle 1990; 10: 224-228

${ }^{7}$ Hertel J. Functional instability following lateral ankle sprain. Sports Med 2000; 29: 361371

${ }^{8}$ Hintermann B. Medial ankle instability. Foot Ankle Clin 2003; 8: 723-738

${ }_{9}^{9}$ Konradsen L. Seven years follow-up after ankle inversion trauma. Scand J Med Sci Sports 2002; 12: 129-135

${ }^{10}$ McCullough CJ, Burge PD. Rotatory stability of the load-bearing ankle: an experimental study. J Bone Joint Surg Am 1980; 62: 460464

11 Mittlmeier T. [Diagnosis and therapy of ligament injuries of the foot]. Unfallchirurg 1998; 101: 137-148

${ }^{12}$ Nicholas R, Hadley J, Paul C et al. "Snowboarder's fracture": fracture of the lateral process of the talus. J Am Board Fam Pract 1994; 7: 130-133

13 Oae K, Takao M, Naito Ket al. Injury of the tibiofibular syndesmosis: value of MR imaging for diagnosis. Radiology 2003; 227: 155-161

14 Ogilvie-Harris DJ, Reed SC, Hedman TP. Disruption of the ankle syndesmosis: biomechanical study of the ligamentous restraints. Arthroscopy 1994: 10: 558-560

15 Outland T. Sprains and separations of inferior tibiofibular joint without important fracture. Am J Surg 1943; 59: 320-329
${ }^{16}$ Rammelt S, Schneiders W, Grass $R$ et al. [Ligamentous injuries to the ankle joint]. Z Orthop Unfall 2011; 149: e45-e67

17 Rasmussen $O$. Stability of the ankle joint: analysis of the function and traumatology of the ankle ligaments. Acta Orthop Scand Suppl 1985; 211: 1-75

18 Richie DH. Functional instability of the ankle and the role of neuromuscular control: a comprehensive review. J Foot Ankle Surg 2001; 40: 240-251

19 Sarrafian SK. Anatomy of the Foot and Ankle: Descriptive, Topographic, Functional. Philadelphia: JB Lippincott; 1983

20 Stiell IG, Greenberg GH, McKnight RD et al. Decision rules for the use of radiography in acute ankle injuries. Refinement and prospective validation. JAMA 1993; 269: 1127-1132

${ }^{21}$ Stormont DM, Morrey BF, An K et al. Stability of the loaded ankle: relation between articular restraint and primary and secondary static restraints. Am J Sports Med 1985; 13: 295 300

Priv.-Doz. Dr. med. Roland Biber Oberarzt

Priv.-Doz. Dr. med. Hermann Josef

Bail

Leitender Arzt

Klinik für Unfall- und Orthopädische Chirurgie

Klinikum Nürnberg - Paracelsus

Medizinische Privatuniversität

Breslauer Straße 201

90471 Nürnberg

biber@klinikum-nuernberg.de 